\title{
Light clusters in dilute heavy-baryon admixed nuclear matter
}

\author{
Armen Sedrakian $^{\left.1,2, \mathrm{a}_{(}\right)}$ \\ ${ }^{1}$ Frankfurt Institute for Advanced Studies, Ruth-Moufang str. 1, 60438 Frankfurt am Main, Germany \\ ${ }^{2}$ Institute of Theoretical Physics, University of Wrocław, pl. M. Borna 9, 50-204 Wrocław, Poland
}

Received: 1 September 2020 / Accepted: 26 September 2020 / Published online: 9 October 2020

(C) The Author(s) 2020

Communicated by David Blaschke

\begin{abstract}
We study the composition of nuclear matter at sub-saturation densities, non-zero temperatures, and isospin asymmetry, under the conditions characteristic of binary neutron star mergers, stellar collapse, and low-energy heavy-ion collisions. The composition includes light clusters with mass number $A \leq 4$, a heavy nucleus $\left({ }^{56} \mathrm{Fe}\right)$, the $\Delta$-resonances, the isotriplet of pions, as well as the $\Lambda$ hyperon. The nucleonic mean-fields are computed from a zero-range density functional, whereas the pion-nucleon interactions are treated to leading order in chiral perturbation theory. We show that with increasing temperature and/or density the composition of matter shifts from light-cluster to heavy baryon dominated one, the transition taking place nearly independent of the magnitude of the isospin. Our findings highlight the importance of simultaneous treatment of light clusters and heavy baryons in the astrophysical and heavy-ion physics contexts.
\end{abstract}

\section{Introduction}

The formation of light clusters in dilute, warm nuclear matter is of interest in astrophysics of binary neutron star mergers, stellar collapse, as well as in heavy-ion physics. The details of the matter composition are important for the accurate determination of transport coefficients appearing in dissipative relativistic fluid dynamics as well as the neutrino Boltzmann transport in various astrophysical scenarios. The clustering phenomenon is also of great interest in nuclear structure calculations (e.g. alpha-clustering) and heavy ion collisions in laboratory experiments.

A great deal of effort during the last decade was focused on the accurate determination of the composition of dilute nuclear matter at finite temperatures and isospin asymmetry within a range of methods based on the ideas of nuclear statistical equilibrium [1-36] and virial expansion for quantum gases [37-40]. The appearance of clusters leads to a range of

a e-mail: sedrakian@ fias.uni-frankfurt.de (corresponding author) interesting phenomena, in particular $\alpha$-condensation at low temperatures [25, 26,30,41-44].

In astrophysics, light clusters and their weak interactions with neutrinos were studied in detail in the context of stellar collapse and supernova physics [45-48]. The electroweak interactions of leptons with baryonic matter are also of interest in describing the transport in binary neutron star mergers, in particular the bulk viscosity [49-52] and electrical conductivity [53,54].

The formation of the heavy baryons in dense and cold nuclear matter, in particular hyperonic members of the $J^{1 / 2+}$ baryonic octet in combinations with the non-strange members of baryon $J^{3 / 2+}$ decouplet ( $\Delta$-resonances) has attracted attention in recent years [55-64]. The relativistic density functionals were successfully tuned to remove the tension between the softening of the equation of state of dense matter associated with the onset of the baryons and the astrophysical observations of the massive neutron stars with masses $2 M_{\odot}[58-60]$.

The motivation of this work is to explore the interplay between the clustering and heavy-baryon degrees of freedom in dilute, finite-temperature nuclear matter. For this purpose we set-up a model which includes both light nuclear clusters with mass number $A \leq 4$, a representative heavy nucleus $\left({ }^{56} \mathrm{Fe}\right)$ as well as the $\Lambda$-hyperon, the quartet of $\Delta$-resonances, and the isotriplet of pions $\pi^{ \pm, 0}$. Previously, hyperons were included in the finite temperature composition of matter in stellar collapse and proto-neutron star studies $[63,65,66]$. Pions and pion condensation has been studied recently in the stellar context in Refs. [66-69]. While the light nuclear clusters have been accounted for in the low-density envelops used in some models, a combined study of the clustering, heavy baryons and pions is missing so far.

In this work, we extend the approach of Ref. [25] to include heavy baryons and pions in the composition and the equation of state of isospin asymmetrical nuclear matter. In addition to the mean-field effects included in the previous study, we will 
treat also the Pauli-blocking effects on the binding energies of the light clusters in an approximate manner. We will focus on temperatures $T \geq 10 \mathrm{MeV}$, which is above the critical temperature of Bose-Einstein condensation of $\alpha$ particles in the clustered environment, see for further details [25,26,30,4244]. Indeed, low temperatures disfavor the heavy baryons in low-density nuclear matter and the problem of $\alpha$ condensation is unaffected by their nucleation. While we include in our composition a heavy nucleus, its effect will turn out to be minor in the parameter range studied in this work.

The paper is organized as follows. Section 2 extends the formalism of the quasiparticle gas model [25] to include heavy baryons and pions. In Sect. 3 we present the numerical results for the composition and equation of state of matter. Section 4 provides a summary and an outlook.

\section{Formalism}

\subsection{Thermodynamics}

We consider matter composed of unbound nucleons, heavy baryons, light nuclei $(A \leq 4),{ }^{56} \mathrm{Fe}$ and pions at temperature $T$ and baryon number density $n_{B}$. We assume that the charge density is fixed to a value $Y_{Q}=n_{L} / n_{B}$, where $n_{L}=\left(n_{e}-\right.$ $\left.n_{e^{+}}\right)+\left(n_{\mu}-n_{\mu^{+}}\right)$where $n_{e}, n_{e^{+}}, n_{\mu}$ and $n_{\mu^{+}}$are the number densities of electrons, positrons, muons and anti-muons. The thermodynamical potential of the system can be expanded into a sum of contributions of constituents

$\Omega\left(\mu_{n}, \mu_{p}, T\right)=\sum_{j} \Omega_{j}\left(\mu_{j}, T\right)$

where $j$ runs over the all elements of the composition of matter, specifically, $j=A, Z$ for nuclei with mass number $A$ and charge $Z, j=n, p$ for neutrons and protons, $j=\left\{\Delta^{0}, \Delta^{+}, \Delta^{++}, \Delta^{-}\right\}$for $\Delta$-resonances, $j=\Lambda$ for the $\Lambda$-hyperon, and $\pi^{0}, \pi^{ \pm}$for the isotriplet of pions. Here the chemical potentials of the species $\mu_{j}$ are functions of the chemical potentials of neutrons and protons $\mu_{n}$ and $\mu_{p}$ in "chemical" equilibrium with respect to weak and strong interactions.

If a nucleus is characterized by mass number $A$ and charge $Z$ its chemical potential is expressed as

$\mu_{A, Z}=(A-Z) \mu_{n}+Z \mu_{p}$.

For the chemical potentials of heavy baryons the following relations hold

$$
\begin{aligned}
\mu_{\Lambda} & =\mu_{\Delta^{0}}=\mu_{n}=\mu_{B}, \\
\mu_{\Delta^{-}} & =2 \mu_{n}-\mu_{p}=\mu_{B}-\mu_{Q}, \\
\mu_{\Delta^{+}} & =\mu_{p}=\mu_{B}+\mu_{Q},
\end{aligned}
$$

$\mu_{\Delta^{++}}=2 \mu_{p}-\mu_{n}=\mu_{B}+2 \mu_{Q}$,

where we introduced the baryon number chemical potential $\mu_{B}$ and the charge chemical potential $\mu_{Q}=\mu_{p}-\mu_{n}$. The chemical potentials of the pions obey the following relations

$\mu_{\pi^{0}}=0$

$\mu_{\pi^{+}}=\mu_{p}-\mu_{n}$,

$\mu_{\pi^{-}}=\mu_{n}-\mu_{p}$.

The baryon number density and the charge neutrality conditions are given by the relations

$$
\begin{aligned}
n_{B}= & n_{n}+n_{p}+\sum_{c} A_{c} n_{c} \\
& +n_{\Delta^{++}}+n_{\Delta^{+}}+n_{\Delta^{-}}+n_{\Delta^{0}}+n_{\Lambda}, \\
n_{B} Y_{Q}= & n_{p}+\sum_{c} Z_{c} n_{c} \\
& +2 n_{\Delta^{++}}+n_{\Delta^{+}}-n_{\Delta^{-}}+n_{\pi^{+}}-n_{\pi^{-}},
\end{aligned}
$$

where the $c$-summation goes over the densities of deuteron $(d)$, triton $(t),{ }^{3} \mathrm{He}(h), \alpha$-particle and ${ }^{56} \mathrm{Fe}$ nucleus. The latter nucleus is considered below in its ground state, i.e., the states that are excited at finite temperatures are neglected. The inclusion of these states will act to enhance the fraction of this particular nucleus or other heavier nuclei in matter, should they be included in the composition. Equations (10) and (11) determine the two unknown chemical potentials $\mu_{n}$ and $\mu_{p}$ at any temperature $T$ for fixed values of $n_{B}$ and $Y_{Q}$.

The thermodynamical potential for each species can be expressed through the densities

$\Omega_{j}\left(\mu_{j}, T\right)=-V \int_{-\infty}^{\mu_{j}} d \mu_{j}^{\prime} n_{j}\left(\mu_{j}^{\prime}, T\right)$,

where $n_{j}\left(\mu_{j}^{\prime}, T\right)$ is the number density of species $j, V$ is the volume.

In the stellar context, the matter is charge neutral, the positive charge of baryons being neutralized by leptons (electrons and muons). The lepton thermodynamic potential is given by

$$
\Omega_{L}=-\sum_{l=e, \mu} g_{l} T \int \frac{d^{3} k}{(2 \pi)^{3}} \ln \left[f_{l}^{-1}\left(-E_{l}(k)+\mu_{l}\right)\right]
$$

where the index $l$ sumes of electrons $e$ and muons $\mu(\tau$ leptons can be neglected), $g_{l}=2$ is the degeneracy factor, the lepton energy is given by $E_{l}=\sqrt{k^{2}+m_{l}^{2}}$, where $m_{l}$ is the lepton mass and $\mu_{l}$ their chemical potential and $f_{l}$ stands for the lepton Fermi distribution function. The lepton density is obtained then as $n_{l}=\partial \Omega_{L} / \partial \mu_{l}$. At finite temperatures a small fraction of positrons may appear: their thermodynamical potential is obtained from Eq. (13) by interchanging the sign of the electron chemical potential. To obtain the full 
thermodynamical potential of matter in astrophysical contexts one needs to take into account, in addition, the thermodynamical potential of neutrinos and anti-neutrinos. For any fixed flavor it has the same form as Eq. (13), the only difference being the degeneracy factor $g_{v}=1$ (as implied by the Standard Model) and vanishingly small neutrino mass.

Having computed partial contributions $\Omega_{j}$, the thermodynamic quantities can be obtained from the thermodynamic potential Eq. (1) for nuclear systems and from the sum of Eqs. (1) and (12) in the charge neutral stellar systems. In particular, we recall that the pressure and the entropy are given by

$P=-\frac{\Omega}{V}, \quad S=-\frac{\partial \Omega}{\partial T}$.

\subsection{Computing densities}

We now turn to the computation of the partial densities of constituents. This can be done in a unified manner for quasiparticles, resonances, and clusters using the real-time finite temperature Green's function (hereafter GF) formalism. The density of species $j$ are directly related to the following GFs

$i G_{j}^{<}\left(x_{1}, x_{2}\right)=\mp\left\langle\psi_{j}^{\dagger}\left(x_{2}\right) \psi_{j}\left(x_{1}\right)\right\rangle$,

$i G_{j}^{>}\left(x_{1}, x_{2}\right)=\left\langle\psi_{j}\left(x_{1}\right) \psi_{j}^{\dagger}\left(x_{2}\right)\right\rangle$,

where $\psi_{j}^{\dagger}\left(x_{1}\right)$ and $\psi_{j}\left(x_{1}\right)$ are the creation and annihilation operators of a species $j$ at the space-time point $x_{1}$, the upper sign here and below refers to fermions, the lower to bosons. The time-arguments of the GF are located on different branches of the Schwinger-Keldysh time-contour with $t_{2}<t_{1}$ in (15) and $t_{2}>t_{1}$ in (16). The Fourier transforms of GFs in (15) and (16) with respect to the argument $x_{1}-x_{2}$ are related to the occupation numbers and the spectral function $S_{j}(\omega, \boldsymbol{p})$ as

$$
\begin{aligned}
-i G_{j}^{<}(\omega, \boldsymbol{p}) & = \pm S_{j}(\omega, \boldsymbol{p}) f(\omega), \\
i G_{j}^{>}(\omega, \boldsymbol{p}) & =S_{j}(\omega, \boldsymbol{p})[1 \mp f(\omega)],
\end{aligned}
$$

where $f_{j}(\omega)$ is either Bose or Fermi distribution function depending on the spin of the $j$-species. From Eqs. (17) and (18) in follows that

$i G_{j}^{>}(\omega, \boldsymbol{p})-i G_{j}^{<}(\omega, \boldsymbol{p})=S_{j}(\omega, \boldsymbol{p})$.

At this point it is convenient to establish the connection to the advanced (A) and retarded (R) GFs

$$
\left[G_{j}^{R / A}(\omega, \boldsymbol{p})\right]^{-1}=\omega-\epsilon_{\boldsymbol{p}}-\Sigma^{R / A}(\omega, \boldsymbol{p}),
$$

where $\epsilon_{p}$ the energy of particle in the non-interacting theory and $\Sigma^{R / A}(\omega, \boldsymbol{p})$ are the retarded/advanced self-energies that are commonly evaluated in the equilibrium theory. If we use the identity

$G_{j}^{>}(\omega, \boldsymbol{p})-G_{j}^{<}(\omega, \boldsymbol{p})=G_{j}^{R}(\omega, \boldsymbol{p})-G_{j}^{A}(\omega, \boldsymbol{p})$,

the spectral function takes the form

$S_{j}(\omega, \mathbf{p})=\frac{\Gamma_{j}(\omega, \mathbf{p})}{\left[\omega-E_{j}(\omega, \mathbf{p})\right]^{2}+\Gamma_{j}^{2}(\omega, \mathbf{p}) / 4}$,

where $E_{j}(\omega, \boldsymbol{p})$ is the quasiparticle energy and $\Gamma_{j}(\omega, \boldsymbol{p})=$ $-2 \operatorname{Im} \Sigma_{j}(\omega, \boldsymbol{p})$ is the spectral width. The quasiparticle energy is given by

$E_{j}(\omega, \boldsymbol{p})=\frac{p^{2}}{2 m_{j}}+E_{j}^{0}+\operatorname{Re} \Sigma_{j}(\omega, \boldsymbol{p})-\mu_{j}$,

where $m_{j}$ is the mass, $E_{j}^{0}$ is the vacuum binding energy of the nucleus $j=(A, Z)$, which vanishes for baryonic quasiparticles. From the definition (15) it follows that

$n_{j}=-i g_{j} \int \frac{d \omega d \boldsymbol{p}}{(2 \pi)^{4}} G_{j}^{<}(\omega, \boldsymbol{p})$,

where $g_{j}$ is the degeneracy factor.

Thus, we have obtained a closed set of equations which consists of Eqs. (10) and (11) for the two unknowns $\mu_{n}$ and $\mu_{p}$ at fixed $n_{B}, Y_{Q}$ and $T$, whereby the densities of constituents are computed from Eqs. (17), (22) (23) and (24). These equations still contain unspecified self-energies of the constituents, which depend on the modeling of the interactions in the system. We turn now to this problem.

\subsection{Self-energies}

We assume that in the dilute limit of interest the unbound baryons are well-defined quasiparticles and the imaginary part of their self-energy vanishes; this implies that their spectral function is a delta-function

$S_{j}(\omega, \boldsymbol{p})=2 \pi \delta\left(\omega-\epsilon_{\boldsymbol{p}, j}-\operatorname{Re} \Sigma_{j}^{R / A}(\omega, \boldsymbol{p})\right)$,

where

$\epsilon_{p, j}=\frac{p^{2}}{2 m_{j}^{*}}-\mu_{j}$

with $m_{j}^{*}$ and $\mu_{j}$ being the effective mass and the chemical potential. Furthermore, the nucleon self-energy is approximated by the effective masses of neutrons $m_{n}^{*}$ and protons $m_{p}^{*}$ which depend on the baryon and charge density (or $n_{B}$ and $Y_{Q}$ ) but are independent of temperature. In the numerical work, we use the Skyrme functional parametrization given by 
Eq. (15) of Ref. [70] for that purpose. The spectral functions of the light clusters are approximated also by their quasiparticle limit

$S_{j}(\omega, \mathbf{p})=2 \pi \delta\left(\omega-\frac{p^{2}}{2 M}-E_{j}^{0}-\operatorname{Re} \Sigma_{j}+\mu_{j}\right)$,

where $E_{j}^{0}$ is the vacuum binding energy of cluster $j, \operatorname{Re} \Sigma_{j}$ is its self-energy. The effective mass of a cluster is constructed as $M=(A-Z) m_{n}^{*}+Z m_{p}^{*}$. Finally, for the $\Lambda$ hyperon and $\Delta$ resonances we use again Eq. (25) with their vacuum masses and neglect the narrow $(118 \mathrm{MeV})$ width of the $\Delta$-resonance and self-energy corrections. With these approximations the energy integral in Eq. (24) is trivial and one is left with the momentum phase-space integration. In the case of pions, we include the leading contribution to the pion self-energy in chiral perturbation theory $[68,71]$, which arises from their coupling to neutrons and protons, specifically Eq. (3.4) and (3.5) in Ref. [71].

The binding energies of clusters are functions of density and temperature in general. The nuclear environment influences the binding energies through phase space occupation (Pauli-blocking). To take this into account, we use the results of the solutions of in-medium two-body Bethe-Salpeter and three-body Faddeev equations in dilute nuclear matter given in Ref. [72]. These solutions are fitted by the following procedure: (a) first we determine the critical value of the inverse temperature $\beta$ for which a cluster disappears via the formula: $\beta_{\mathrm{cr}}\left[\mathrm{MeV}^{-1}\right]=0.07835+0.00185\left(n_{0} / n_{B}\right)$, where $n_{0}=0.16 \mathrm{fm}^{-3}$, which is assumed to be universally independent of $A$ and $Y_{Q}$, and (b) the in-medium binding energies $B_{j}\left(n_{B}, T\right)$ are obtained via a linear fit given by

$B_{j}\left(n_{B}, T\right)=E_{j}^{0}\left[1-\frac{\beta}{\beta_{\mathrm{cr}}\left(n_{0} / n_{B}\right)}\right]$.

Then the spectral function (25) takes the form

$S_{j}(\omega, \boldsymbol{p}) \simeq 2 \pi \delta\left(\omega-\frac{p^{2}}{2 M}-B_{j}+\mu_{j}^{*}\right)$,

where any contribution to the self-energy beyond the modifications of the binding energy is energy and momentum independent and, thus, can be absorbed in the chemical potential $\mu_{j}^{*}$.

\section{Results}

The system of Eqs. (10) and (11) was solved simultaneously for unknown chemical potentials $\mu_{n}$ and $\mu_{p}$ at fixed temperature $T$, baryon number density $n_{B}$ and charge fraction $Y_{Q}$. We consider two values of the latter parameter $Y_{Q}=0.1$,

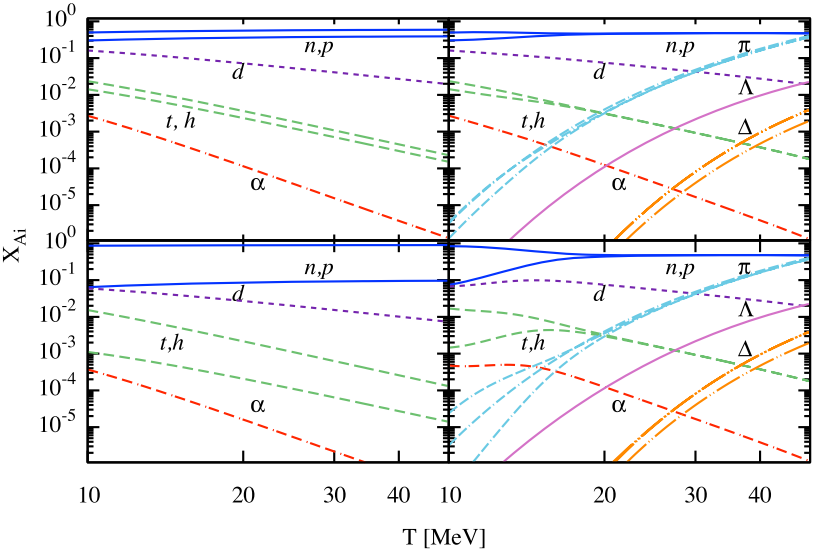

Fig. 1 Dependence of the mass fractions of the particles in dilute nuclear matter on temperature at constant density $n_{B} / n_{0}=10^{-2}$. The top and lower panels correspond to charge fractions $Y_{Q}=0.4$ and 0.1 . The left and right panels correspond to the cases containing nucleons and light clusters only and the full composition, respectively. The composition includes neutrons and protons (solid lines), deuterons (shortdashed), triton and helium (long-dashed), $\alpha$-particles (dash-dotted), $\Delta$ resonances (dash-double-dot), $\Lambda$-hyperon (dash-triple-dot), and pions (double-dash-dot). The mass fraction of ${ }^{56} \mathrm{Fe}$ is not visible on the figure's scale

which is characteristic to binary neutron star mergers, and $Y_{Q}=0.4$ which is characteristic to stellar collapse.

Figure 1 shows the mass fraction $X_{j}=A_{j} n_{j} / n_{B}$, where $A_{j}$ is the mass number of a constituent, as a function of temperature in cases (a) nucleons and clusters only and (b) nucleons, clusters, heavy baryons and pions, for $Y_{Q}=0.1$ and 0.4 at fixed $n_{B} / n_{0}=10^{-2}$, where $n_{0}=0.16 \mathrm{fm}^{-3}$ is the nuclear saturation density. The mass fraction of ${ }^{56} \mathrm{Fe}$ is not visible on figure's scale. It is seen that nucleons are the dominant component at all temperatures, but there is a change in the composition of matter with respect to the remaining constituents with increasing temperature. For temperatures $T \geq 30 \mathrm{MeV}$ the dominant mass fraction is in the heavy baryons, whereas at lower temperatures the clusters are the dominant component. Note also that the inclusion of heavy baryons and pions reduces the isospin asymmetry in the neutron and proton components and, as a consequence, the helion and triton abundances are much closer to each other in this case. A previous study of hyperon abundances at finite temperatures in Ref. [28] finds that the hyperon fraction exceeds $10^{-4}$ at density $n_{B} / n_{0}=10^{-2}$ for temperatures $T \geq 40 \mathrm{MeV}$. According to Fig. 1 this occurs in our model for $T \geq 20 \mathrm{MeV}$. This difference may be a consequence of different treatment of nuclear interactions and different compositions allowed in the models. Ref. [27] finds that $\Lambda$ hyperon fraction stays below $10^{-7}$ for temperatures up to $14 \mathrm{MeV}$ in the inhomogeneous "pasta" phases of supernova matter independent of the value of $Y_{Q}$, which is consistent with present results. 


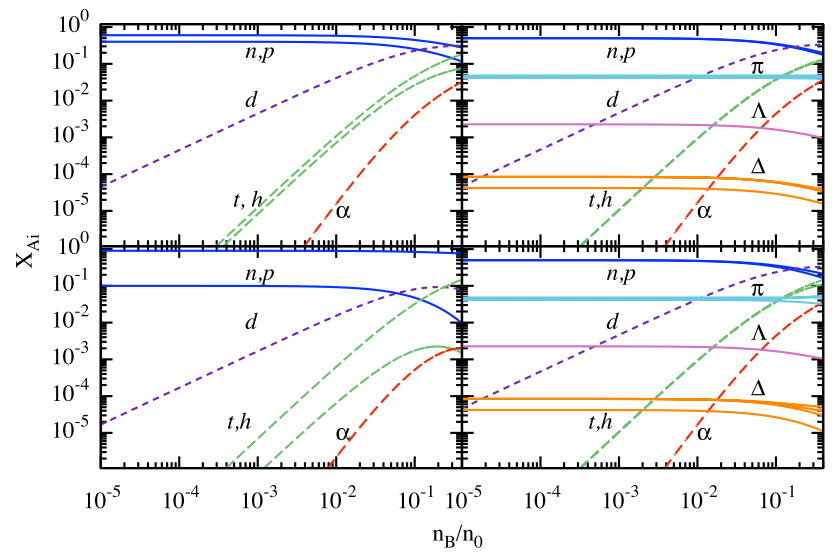

Fig. 2 Dependence of the mass fractions of the particles in dilute nuclear matter on density for $T=30 \mathrm{MeV}$. The top and lower panels correspond to charge fraction $Y_{Q}=0.4$ and 0.1 and the left and right panels correspond to the cases containing only nucleons and light clusters and the full composition, respectively. The composition includes neutrons and protons (solid lines), deuterons (short-dashed), triton and helium (long-dashed), $\alpha$-particles (dash-dotted), $\Delta$ resonances (dashdouble-dot), $\Lambda$-hyperon (dash-triple-dot), and pions (double-dash-dot). In the right figure, the clusters disappear for $n_{B} / n_{0} \geq 9 \times 10^{-2}$ (shaded area) due to the Pauli-blocking of the phase-space. The mass fraction of ${ }^{56} \mathrm{Fe}$ is not visible on the figure's scale

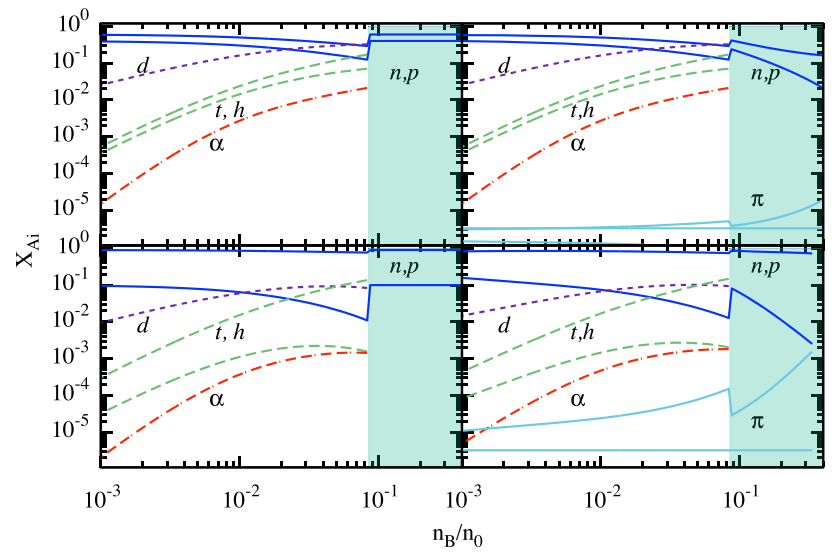

Fig. 3 Same as in Fig. 2 but for $T=10 \mathrm{MeV}$

Figures 2 and 3 show the mass fractions mass fraction $X_{j}$ at two fixed temperatures $T=30 \mathrm{MeV}$ and $T=10 \mathrm{MeV}$ and varying density. It is seen that the abundances of the nucleons, heavy baryons, and pions are insensitive to the density, whereas the cluster abundances increase as the density increases. In other words, the increase in the nucleonic density at a fixed temperature is accommodated by the system by increasing the number of the light clusters, whereas the fractions of neutrons and protons remain constant in a wide density range. Since the heavy baryon fraction are determined by their "chemical" equilibrium with respect to neutrons and protons via the relations (3)-(6), their fractions

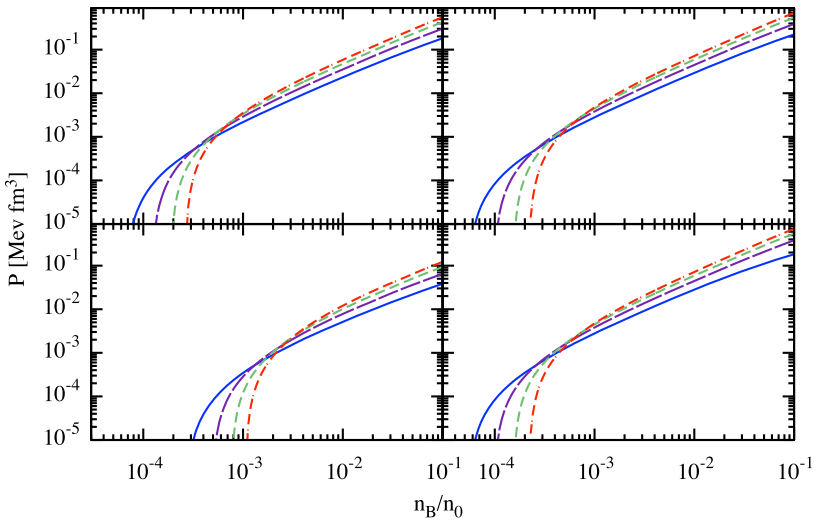

Fig. 4 Pressure as a function of normalized density $n_{B} / n_{0}$ for temperature values (in MeV) $T=20$ (solid lines), 30 (long-dashed), 40 (short-dashed), and 50 (dash-doted). The upper panels correspond to $Y_{Q}=0.4$ and the lower ones - to $Y_{Q}=0.1$. The composition in panels is as in Fig. 1

stay constant with the density as well. The same applies also to pion fractions, which are likewise related to proton and neutron concentrations via Eqs. (8) and (9). The reduction of isospin asymmetry among neutrons and protons mentioned above is seen here as well. Note that the Pauliblocking at $T=30 \mathrm{MeV}$ is ineffective within the density range considered, but its effect is seen in the right panels of Fig. 3 corresponding to $T=10 \mathrm{MeV}$. It is seen that $n_{B} / n_{0} \simeq 0.1$ the clusters abruptly disappear as a consequence of $B_{j}\left(n_{B}, T\right) \rightarrow 0$ and there appears a jump in the density of nucleons. Note that our Pauli blocking factor does not dependent on the momentum of the cluster with respect to the medium. In general, it does, so that the phase space vanishes with increasing the density more smoothly: the clusters with the lower-momenta are eliminated first, while those with high-momenta remain intact. It is also seen that the pion mass fraction undergoes at the same point an abrupt change, clearly visibly for $Y_{Q}=0.1$. Finally, note that at this temperature, the heavy baryon fractions are too low to be relevant. To assess if there is a phase transition (and if so, to find its order) a detailed study of the thermodynamic functions of matter at the point of the dissolution of clusters is needed. In a similar study of Ref. [6], which used non-linear fits to the binding energies of clusters, the transition is found to be less abrupt.

Figure 4 shows the pressure as a function of the normalized density for temperature values $T=20,30,40$, and $50 \mathrm{MeV}$ for two values of charge fraction $Y_{Q}=0.1$ and 0.4 . The main effect caused by the onset of heavy baryons and pions is the more symmetric appearance of the nucleonic component for $Y_{Q}=0.1$, already observed in Fig. 2, which leads to pressure values that are similar to those for the case $Y_{Q}=0.4$. 


\section{Summary and outlook}

The composition of warm dilute nuclear matter was computed including simultaneously light clusters with $A \leq 4$, a representative heavy nucleus $\left({ }^{56} \mathrm{Fe}\right)$, heavy baryons $(\Lambda$ 's and $\Delta$ 's) and pions. We find that with increasing temperature the mass fraction shifts from light clusters to heavy baryons, whereby the nucleons remain the dominant component within the parameter range considered. The heavy nucleus ${ }^{56} \mathrm{Fe}$ does not play a significant role at temperatures $T \geq 10 \mathrm{MeV}$, but is known to suppress strongly the abundances of light clusters at low temperatures of the order $1 \mathrm{MeV}[25,44]$. The addition of heavy baryons and pions makes the nucleonic component more isospin symmetric and, as a consequence, the cluster abundances become less sensitive to the value of the isospin asymmetry. At low temperatures $T \simeq 10 \mathrm{MeV}$, the phase-space occupation strongly suppresses the cluster abundances for densities $n_{B} / n_{0} \geq 0.1$ due to the Pauli blocking, as expected.

The rich composition of matter in the parameter range considered may have interesting implications in astrophysics of compact star mergers, stellar collapse as well as heavyion collisions. The transport studies of hadronic matter and its coupling to leptons in these contexts need to include the additional degrees of freedom shown to be important in the composition of matter.

Acknowledgements The support through the European COST Actions "PHAROS" (CA16214) and by the Deutsche Forschungsgemeinschaft (Grant no. SE 1836/5-1) is gratefully acknowledged. This research has made use of NASA's Astrophysics Data System Bibliographic Services.

Data Availability Statement This manuscript has no associated data or the data will not be deposited. [Authors' comment: Data are available on request from the author.]

Open Access This article is licensed under a Creative Commons Attribution 4.0 International License, which permits use, sharing, adaptation, distribution and reproduction in any medium or format, as long as you give appropriate credit to the original author(s) and the source, provide a link to the Creative Commons licence, and indicate if changes were made. The images or other third party material in this article are included in the article's Creative Commons licence, unless indicated otherwise in a credit line to the material. If material is not included in the article's Creative Commons licence and your intended use is not permitted by statutory regulation or exceeds the permitted use, you will need to obtain permission directly from the copyright holder. To view a copy of this licence, visit http://creativecomm ons.org/licenses/by/4.0/.

\section{References}

1. K. Sumiyoshi, G. Röpke, Appearance of light clusters in postbounce evolution of core-collapse supernovae. Phys. Rev. C 77, 055804 (2008). arXiv:0801.0110

2. C. Ducoin, J. Margueron, P. Chomaz, Cluster formation in asymmetric nuclear matter: semi-classical and quantal approaches. Nucl. Phys. A 809, 30-64 (2008). arXiv:0801.2660
3. A. Arcones, G. Martínez-Pinedo, E. O'Connor, A. Schwenk, H.-T. Janka, C.J. Horowitz et al., Influence of light nuclei on neutrinodriven supernova outflows. Phys. Rev. C 78, 015806 (2008). arXiv:0805.3752

4. S. Heckel, P.P. Schneider, A. Sedrakian, Light nuclei in supernova envelopes: a quasiparticle gas model. Phys. Rev. C 80, 015805 (2009). arXiv:0902.3539

5. S.R. Souza, A.W. Steiner, W.G. Lynch, R. Donangelo, M.A. Famiano, Comparison of statistical treatments for the equation of state for core-collapse supernovae. ApJ 707, 1495-1505 (2009). arXiv:0810.0963

6. S. Typel, G. Röpke, T. Klähn, D. Blaschke, H.H. Wolter, Composition and thermodynamics of nuclear matter with light clusters. Phys. Rev. C 81, 015803 (2010). arXiv:0908.2344

7. A.S. Botvina, I.N. Mishustin, Statistical approach for supernova matter. Nucl. Phys. A 843, 98-132 (2010). arXiv:0811.2593

8. A.R. Raduta, F. Gulminelli, Statistical description of complex nuclear phases in supernovae and proto-neutron stars. Phys. Rev. C 82, 065801 (2010). arXiv: 1009.2226

9. M. Hempel, J. Schaffner-Bielich, A statistical model for a complete supernova equation of state. Nucl. Phys. A 837, 210-254 (2010). arXiv:0911.4073

10. G. Röpke, Parametrization of light nuclei quasiparticle energy shifts and composition of warm and dense nuclear matter. Nucl. Phys. A 867, 66-80 (2011). arXiv:1101.4685

11. M. Hempel, J. Schaffner-Bielich, S. Typel, G. Röpke, Light clusters in nuclear matter: excluded volume versus quantum many-body approaches. Phys. Rev. C 84, 055804 (2011). arXiv:1109.0252

12. L. Qin, K. Hagel, R. Wada, J.B. Natowitz, S. Shlomo, A. Bonasera et al., Laboratory tests of low density astrophysical nuclear equations of state. Phys. Rev. L 108, 172701 (2012). arXiv:1110.3345

13. M. Hempel, T. Fischer, J. Schaffner-Bielich, M. Liebendörfer, New equations of state in simulations of core-collapse supernovae. ApJ 748, 70 (2012). arXiv: 1108.0848

14. F. Gulminelli, A.R. Raduta, Ensemble inequivalence in supernova matter within a simple model. Phys. Rev. C 85, 025803 (2012). arXiv: 1110.2034

15. M. Ferreira, C. Providência, Description of light clusters in relativistic nuclear models. Phys. Rev. C 85, 055811 (2012). arXiv: 1206.0139

16. A.R. Raduta, F. Aymard, F. Gulminelli, Clusterized nuclear matter in the (proto-)neutron star crust and the symmetry energy. Eur. Phys. J. A 50, 24 (2014). arXiv: 1307.4202

17. N. Buyukcizmeci, A.S. Botvina, I.N. Mishustin, Tabulated equation of state for supernova matter including full nuclear ensemble. ApJ 789, 33 (2014). arXiv: 1304.6741

18. F. Aymard, F. Gulminelli, J. Margueron, In-medium nuclear cluster energies within the extended Thomas-Fermi approach. Phys. Rev. C 89, 065807 (2014). arXiv:1404.3096

19. F. Gulminelli, A.R. Raduta, Unified treatment of subsaturation stellar matter at zero and finite temperature. Phys. Rev. C 92, 055803 (2015). arXiv: 1504.04493

20. M. Hempel, K. Hagel, J. Natowitz, G. Röpke, S. Typel, Constraining supernova equations of state with equilibrium constants from heavy-ion collisions. Phys. Rev. C 91, 045805 (2015). arXiv: 1503.00518

21. S. Burrello, F. Gulminelli, F. Aymard, M. Colonna, A.R. Raduta, Heat capacity of the neutron star inner crust within an extended nuclear statistical equilibrium model. Phys. Rev. C 92, 055804 (2015)

22. G. Röpke, Nuclear matter equation of state including two-, three-, and four-nucleon correlations. Phys. Rev. C 92, 054001 (2015). arXiv: 1411.4593

23. S. Furusawa, I. Mishustin, Self-consistent calculation of the nuclear composition in hot and dense stellar matter. Phys. Rev. C 95, 035802 (2017). arXiv:1612.01854 
24. S.S. Avancini, M. Ferreira, H. Pais, C. Providência, G. Röpke, Light clusters and pasta phases in warm and dense nuclear matter. Phys. Rev. C 95, 045804 (2017). arXiv: 1704.00054

25. X.H. Wu, S.-B. Wang, A. Sedrakian, G. Röpke, Composition of nuclear matter with light clusters and Bose-Einstein condensation of $\alpha$ particles. J. Low Temp. Phys. 189, 133-146 (2017). arXiv: 1705.02525

26. Z.-W. Zhang, L.-W. Chen, Low density nuclear matter with light clusters in a generalized nonlinear relativistic mean-field model. Phys. Rev. C 95, 064330 (2017). arXiv:1705.00555

27. D.P. Menezes, C. Providência, Hyperons in the nuclear pasta phase. Phys. Rev. C 96, 045803 (2017). arXiv:1707.01338

28. M. Fortin, M. Oertel, C. Providência, Hyperons in hot dense matter: what do the constraints tell us for equation of state? PASA 35, 44 (2018). arXiv: 1711.09427

29. H. Pais, F. Gulminelli, C. Providência, G. Röpke, Light clusters in warm stellar matter: explicit mass shifts and universal cluster-meson couplings. Phys. Rev. C 97, 045805 (2018). arXiv: 1804.01328

30. Z.-W. Zhang, L.-W. Chen, Cold dilute nuclear matter with $\alpha$ particle condensation in a generalized nonlinear relativistic meanfield model. Phys. Rev. C 100, 054304 (2019). arXiv:1903.04108

31. G. Grams, S. Giraud, A.F. Fantina, F. Gulminelli, Distribution of nuclei in equilibrium stellar matter from the free-energy density in a Wigner-Seitz cell. Phys. Rev. C 97, 035807 (2018)

32. A.R. Raduta, F. Gulminelli, Nuclear statistical equilibrium equation of state for core collapse. Nucl. Phys. A 983, 252-275 (2019). arXiv: 1807.06871

33. H. Pais, F. Gulminelli, C. Providência, G. Röpke, Full distribution of clusters with universal couplings and in-medium effects. Phys. Rev. C 99, 055806 (2019). arXiv: 1904.10373

34. G. Röpke, Light $\mathrm{p}$-shell nuclei with cluster structures $(4 \leq \mathrm{A} \leq 16)$ in nuclear matter. Phys. Rev. C 101, 064310 (2020). [2004.09773]

35. H. Pais, R. Bougault, F. Gulminelli, C. Providência, E. Bonnet, B. Borderie et al., Low density in-medium effects on light clusters from heavy-ion data. Phys. Rev. L 125, 012701 (2020). arXiv: 1911.10849

36. S. Mallik, F. Gulminelli, Statistical treatment of nuclear clusters in the continuum. (2020). arXiv:2008.11371

37. M. Schmidt, G. Röpke, H. Schulz, Generalized Beth-Uhlenbeck approach for hot nuclear matter. Ann. Phys. 202, 57-99 (1990)

38. A. Sedrakian, G. Röpke, A quantum kinetic equation for fermi systems including three-body correlations. Ann. Phys. 266, 524550 (1998). arXiv:nucl-th/9712074

39. C.J. Horowitz, A. Schwenk, Cluster formation and the virial equation of state of low-density nuclear matter. Nucl. Phys. A 776, 55-79 (2006). arXiv:nucl-th/0507033

40. S. Mallik, J.N. de, S.K. Samaddar, S. Sarkar, S-matrix approach to equation of state of nuclear matter. Phys. Rev. C 77, 032201 (2008). arXiv:0801.0498

41. A. Sedrakian, H. Müther, P. Schuck, Alpha matter on a lattice. Nucl. Phys. A 766, 97-106 (2006). arXiv:nucl-th/0407020

42. L.M. Satarov, M.I. Gorenstein, A. Motornenko, V. Vovchenko, I.N. Mishustin, H. Stoecker, Bose-Einstein condensation and liquidgas phase transition in strongly interacting matter composed of $\alpha$ particles. J. Nucl. Phys. G 44, 125102 (2017). arXiv:1704.08039

43. L.M. Satarov, I.N. Mishustin, A. Motornenko, V. Vovchenko, M.I. Gorenstein, H. Stoecker, Phase transitions and Bose-Einstein condensation in $\alpha$-nucleon matter. Phys. Rev. C 99, 024909 (2019). arXiv: 1811.02924

44. S. Furusawa, I. Mishustin, Degeneracy effects and Bose condensation in warm nuclear matter with light and heavy clusters. Nucl. Phys. A 1002, 121991 (2020)

45. T. Fischer, M. Hempel, I. Sagert, Y. Suwa, J. Schaffner-Bielich, Symmetry energy impact in simulations of core-collapse supernovae. Eur. Phys. J. A 50, 46 (2014). arXiv:1307.6190
46. T. Fischer, G. Martínez-Pinedo, M. Hempel, L. Huther, G.Röpke, S. Typel et al., Expected impact from weak reactions with light nuclei in corecollapse supernova simulations, in European Physical Journal Web of Conferences, vol. 109 of European Physical Journal Web of Conferences, p. 06002 (2016). arXiv:1512.00193

47. H. Nagakura, S. Furusawa, H. Togashi, S. Richers, K. Sumiyoshi, S. Yamada, Comparing treatments of weak reactions with nuclei in simulations of core-collapse supernovae. ApJS 240, 38 (2019). arXiv: 1812.09811

48. T. Fischer, S. Typel, G.Röpke, N.-U.F. Bastian, G. MartínezPinedo, Medium modifications for light and heavy nuclear clusters in simulations of core collapse supernovae-impact on equation of state and weak interactions. (2020). arXiv:2008.13608

49. M.G. Alford, S.P. Harris, Damping of density oscillations in neutrino-transparent nuclear matter. Phys. Rev. C 100, 035803 (2019). arXiv:1907.03795

50. M. Alford, A. Harutyunyan, A. Sedrakian, Bulk viscosity of baryonic matter with trapped neutrinos. Phys. Rev. D 100, 103021 (2019). arXiv:1907.04192

51. M. Alford, A. Harutyunyan, A. Sedrakian, Bulk viscous damping of density oscillations in neutron star mergers. Particles 3, 500-517 (2020)

52. M.G. Alford, A. Haber, Hyperon bulk viscosity in neutron star mergers. (2020). arXiv:2009.05181

53. A. Harutyunyan, A. Sedrakian, Electrical conductivity of a warm neutron star crust in magnetic fields. Phys. Rev. C 94, 025805 (2016). arXiv:1605.07612

54. A. Harutyunyan, A. Nathanail, L. Rezzolla, A. Sedrakian, Electrical resistivity and Hall effect in binary neutron star mergers. Eur. Phys. J. A 54, 191 (2018). arXiv: 1803.09215

55. A. Drago, A. Lavagno, G. Pagliara, D. Pigato, Early appearance of $\Delta$ isobars in neutron stars. Phys. Rev. C 90, 065809 (2014)

56. B.-J. Cai, F.J. Fattoyev, B.-A. Li, W.G. Newton, Critical density and impact of $\Delta(1232)$ resonance formation in neutron stars. Phys. Rev. C 92, 015802 (2015)

57. H.S. Sahoo, G. Mitra, R. Mishra, P.K. Panda, B.-A. Li, Neutron star matter with $\Delta$ isobars in a relativistic quark model. Phys. Rev. C 98, 045801 (2018)

58. E. Kolomeitsev, K. Maslov, D. Voskresensky, Delta isobars in relativistic mean-field models with $\sigma$-scaled hadron masses and couplings. Nucl. Phys. A 961, 106-141 (2017)

59. J.J. Li, A. Sedrakian, F. Weber, Competition between delta isobars and hyperons and properties of compact stars. Phys. Lett. B 783, 234-240 (2018)

60. J.J. Li, A. Sedrakian, Implications from GW170817 for $\Delta$-isobar admixed hypernuclear compact stars. ApJ Lett. 874, L22 (2019). arXiv: 1904.02006

61. J.J. Li, A. Sedrakian, M. Alford, Relativistic hybrid stars with sequential first-order phase transitions and heavy-baryon envelopes. Phys. Rev. D 101, 063022 (2020)

62. P. Ribes, A. Ramos, L. Tolos, C. Gonzalez-Boquera, M. Centelles, Interplay between $\Delta$ particles and hyperons in neutron stars. ApJ 883, 168 (2019). arXiv: 1907.08583

63. A.R. Raduta, M. Oertel, A. Sedrakian, Proto-neutron stars with heavy baryons and universal relations. (2020). arXiv:2008.00213

64. A. Sedrakian, F. Weber, J.-J. Li, Confronting GW190814 with hyperonization in dense matter and hypernuclear compact stars. Phys. Rev. D 102, 041301 (2020). arXiv:2007.09683

65. K. Nakazato, S. Furusawa, K. Sumiyoshi, A. Ohnishi, S. Yamada, H. Suzuki, Hyperon matter and black hole formation in failed supernovae. ApJ 745, 197 (2012). arXiv:1111.2900

66. B. Peres, M. Oertel, J. Novak, Influence of pions and hyperons on stellar black hole formation. Phys. Rev. D 87, 043006 (2013). arXiv: 1210.7435 
67. C. Ishizuka, A. Ohnishi, K. Tsubakihara, K. Sumiyoshi, S. Yamada, Tables of hyperonic matter equation of state for core-collapse supernovae. J. Nucl. Phys. G 35, 085201 (2008). arXiv:0802.2318

68. G. Colucci, E.S. Fraga, A. Sedrakian, Chiral pions in a magnetic background. Phys. Lett. B 728, 19-24 (2014). arXiv: 1310.3742

69. B. Fore, S. Reddy, Pions in hot dense matter and their astrophysical implications. Phys. Rev. C 101, 035809 (2020). arXiv:1911.02632

70. D. Davesne, A. Pastore, J. Navarro, Extended Skyrme equation of state in asymmetric nuclear matter. A \& A 585, A83 (2016). arXiv: 1509.05744
71. J.A. Oller, A. Lacour, U.G. Meißner, Chiral effective field theory for nuclear matter with long- and short-range multi-nucleon interactions. J. Nucl. Phys. G 37, 015106 (2010). arXiv:0902.1986

72. A. Sedrakian, J.W. Clark, Pair condensation and bound states in fermionic systems. Phys. Rev. C 73, 035803 (2006). arXiv:nucl-th/0511076 